\title{
Folksonomia: esquema de representação do conhecimento?
}

\author{
Folksonomy: knowledge representation system?
}

Mariana BRANDT'

Marisa Brascher Basílio MEDEIROS²

\section{RES U M O}

O artigo tem como objetivo estudar a folksonomia sob a óptica da representação do conhecimento. Para isso, apoia-se em revisão bibliográfica das abordagens em organização do conhecimento propostas por Hiorland, nas quais a folksonomia encontra bases para ser analisada: abordagem baseada no usuário, cognitiva, social e da recuperação da informação. Traz uma breve discussão sobre organização do conhecimento e organização da informação, definindo como tais conceitos serão tratados no artigo. Ressalta os pontos positivos e negativos da folksonomia como esquema de representação do conhecimento, analisando também como essa estrutura pode ou não refletir conhecimento. Identifica a folksonomia como um sistema construído de forma inversa em relação aos outros sistemas, em que a coleta de termos e estruturação é feita a posteriori. Enquadra a folksonomia nos esquemas de representação, no sentido de ser vista como ferramenta semântica. Propõe novos tipos de estudos que comparem a folksonomia com os esquemas tradicionais de representação do conhecimento, como tesauros, taxonomias e ontologias.

Palavras-chave: Folksonomia. Organização do conhecimento. Organização da informação. Representação do conhecimento. Web.

\section{A B S T R A C T}

The article aims to study folksonomy in the view of knowledge representation. A bibliographic review of the approaches to knowledge organization proposed by Hiorland is conducted in order to find out how folksonomy can be matched. The approaches are: user-based, cognitive, social and information retrieval approach. The article also includes a brief discussion about knowledge organization and information organization, defining how those concepts will be addressed in the article. It highlights the positive and negative aspects of folksonomy as a knowledge representation scheme, and it also analyzes how it can reflect or fail to reflect knowledge. In addition, it identifies folksonomy as a system designed differently than other systems, in which term collections and structure is done a posteriori. It sets folksonomy in the representation schemes, in order to be considered a semantic tool. It suggests new studies to compare folksonomy to the traditional knowledge representation schemes, such as thesauri, taxonomies and ontology.

Keywords: Folksonomy. Knowledge organization. Knowledge representation. Information organization. Web.

1 Professora, Universidade de Brasília, Faculdade de Ciência da Informação, Programa de Pós-Graduação em Ciência da Informação. Anexo à Biblioteca Central da UnB, Campus Universitário Darcy Ribeiro, 70919-970, Brasília, DF, Brasil. Correspondência para/Correspondence to: M. BRANDT.E-mail: <marianabrandt@gmail.com>.

2 Professora Doutora, Universidade de Brasília, Faculdade de Ciência da Informação, Departamento de Ciência da Informação e Documentação. Brasília, DF, Brasil.

Recebido em 19/4/2010 e aceito para publicação em 7/7/2010. 


\section{N TR O D U Ç Ã O}

Folksonomia é o resultado do processo de etiquetagem, também chamado de classificação social, de recursos da web. Isso significa dizer que as próprias pessoas, no caso, usuários da informação, classificam os documentos. O termo surgiv em 2004 no contexto da Internet, onde a observação de uma prática crescente - a atribuição de etiquetas (tags) a conteúdos informacionais, despertou o interesse de pessoas que acompanham os fenômenos que ocorrem na web. Tal interesse suscitou um tópico sobre o assunto em uma lista de discussão e daí uma necessidade de denominação de tal fenômeno.

A criação do termo folksonomia é atribuída ao arquiteto da informação Thomas Vander Wal, que o define como:

[...] o resultado da atribuição livre e pessoal de etiquetas a informações ou objetos (qualquer coisa com URL), visando à sua recuperação. $A$ atribuição de etiquetas é feita num ambiente social (compartilhado e aberto a outros). A etiquetagem é feita pelo próprio consumidor da informação (Wal, 2007, online).

Segundo o autor, o termo vem da sugestão de outro membro da lista, Eric Scheid: folk classification (classificação por pessoas, ou social). O prefixo folk é então usado por Wal para substituir o 'tax', de taxonomia, gerando o termo folksonomia. Considerando questões etimológicas, o termo é congruente, iá que elimina o tax (regra) da taxonomia: a folksonomia é uma atribuição livre e pessoal de etiquetas, ou seja, livre das regras impostas por uma taxonomia.

Relata-se na literatura que os primeiros sites a permitirem que seus usuários atribuíssem etiquetas aos recursos informacionais foram o Del.icio.us ${ }^{\mathrm{TM}}$ (http:// del.icio.us/), em 2003 e posteriormente o Flickr ${ }^{\text {TM }}$ (http:/ /www.flickr.com/). Em pouco tempo, vários outros serviços de informação na web passaram a adotar tal prática. Com isso, a folksonomia vem sendo uma aclamada implementação da chamada Web 2.0. Segundo O'Reilly (2005), as taxonomias tradicionais e estruturas em diretório são características da Web 1.0, enquanto a folksonomia se enquadra no contexto de compartilhamento proposto pela Web 2.0.

Este artigo pretende analisar a folksonomia sob a óptica dos esquemas de representação do conheci- mento de acordo com as abordagens indicadas por Hiørland (2007a), identificando possíveis características que permitam enquadrá-la, ou não, como tal.

\section{ORGANIZAÇÃO DO CONHECIMEN- TO VERSUS ORGANIZAÇÃO DA IN- FORMAÇ Ã O}

Para se discutir organização do conhecimento e da informação deve-se, primeiramente, fazer uma breve distinção dos conceitos "conhecimento" e "informação". Há muita discussão sobre o tema, mas para fins desta análise, entende-se conhecimento como o conjunto de conceitos (unidades do conhecimento) presentes em determinada área temática, e informação como o registro físico desse conhecimento. A partir daí, pode-se entender organização do conhecimento e organização da informação.

Para Dahlberg (2006), citado por Hiørland (2007c, tradução nossa), organização do conhecimento

[...] é a ciência de construir e arranjar sistematicamente unidades do conhecimento (conceitos) de acordo com seus elementos do conhecimento (características) inerentes e a aplicação de conceitos e classes de conceitos ordenados por sua forma de atribuição de conteúdos de referência válidos (objetos/sujeitos/assuntos) de todos os tipos.

Pode-se entender, então, que a organização do conhecimento está relacionada com um processo de análise conceitual de um domínio do conhecimento, e, a partir daí, sua estruturação, gerando uma representação do conhecimento de tal domínio. Dessa forma, obtém-se um instrumento - um esquema de representação do conhecimento - que será então usado para a organização da informação desse domínio de conhecimento produzida.

Taylor (2003) também faz essa discussão em relação ao que é organizado. Para a autora, organizamos informação quando a organizamos para o benefício de outras pessoas, ou seja, organizamos os objetos que contém informação. Taylor denomina tais objetos como objetos informacionais: unidades de informação organizável. Para este trabalho, optou-se por utilizar a denominação objeto informacional, como proposto por Bräscher e Café (2008). 
Dessa forma, tem-se a distinção entre organização do conhecimento e organização da informação: a partir da organização do conhecimento de um domínio, como definido por Dalhberg, pode-se organizar a informação, ou os objetos informacionais produzidos neste domínio, como entendido por Taylor. Para tanto, utilizam-se os esquemas de organização ou representação do conhecimento. De forma ampla, podem-se considerar os objetos informacionais como as unidades de organização do conhecimento. Há, porém, autores como Broughton et al. (2005) que afirmam que para cada abordagem em Organização do conhecimento, há uma unidade diferente a ser organizada.

Já para Hodge (2000), o termo sistema de organização do conhecimento pretende englobar todos os tipos de esquemas para organização da informação e promover o gerenciamento do conhecimento. A autora utiliza o termo de acordo com a definição cunhada pela Network Knowledge Organization Work Group em seu primeiro encontro em 1998.

Hiørland (2007c) discute que a definição de Dalhberg (1993) remete a um processo, mas defende que o estudo da organização do conhecimento se dá em duas vertentes: os processos de organização do conhecimento e os sistemas de organização do conhecimento. Para Broughton et al. (2005), no sentido estrito, organização do conhecimento se refere aos sistemas de organização do conhecimento como registros bibliográficos, sistemas de classificação, redes semânticas e tesauros. Já os processos de organização do conhecimento, segundo os autores, são a catalogação, a classificação, a indexação, e a análise de assunto. Tais processos são entendidos por Taylor como organização da informação, e não do conhecimento, conforme citado anteriormente.

Tem-se, então, que o termo "organização do conhecimento" é entendido por alguns autores como um "processo" (que para outros autores seria organização da informação). Este processo pode utilizar como auxílio para sua execução uma ferramenta elaborada para a "representação do conhecimento", ou seja, um esquema de representação do conhecimento - que é a outra vertente da disciplina "Organização do conhecimento", nessa perspectiva. Desta forma, acredita-se que a denominação "esquema de representação do conhecimento" seja mais apropriada e menos ambígua, sendo, portanto, adotada neste trabalho.
Tal denominação é também utilizada por Sowa (2000). O autor afirma que representação do conhecimento é um assunto multidisciplinar que aplica teorias e técnicas de três outras áreas: lógica, ontologia e computação. A representação do conhecimento, para este autor, é a aplicação da lógica e da ontologia nas tarefas de construção de modelos computadorizados para algum domínio. Essa visão é, de certa forma, restrita para a análise proposta neste trabalho.

Muito antes disso, Vickery (1986) também usa o termo "representação do conhecimento", e fala que essa vem sendo uma questão que preocupa o mundo da documentação desde sua origem. Afirma ainda que a questão passou a ser preocupação também de várias outras áreas como computação, linguagem, inteligência artificial e psicologia. Em todas essas áreas é necessário definir como o conhecimento vai ser representado para que as representações permitam sua manipulação. Para Vickery (1986, p. 145):

Muitas técnicas diferentes de representação do
conhecimento vêm sendo desenvolvidas em
cada campo, as variações costumam se dar de
acordo com os diferentes tipos de manipula-
ção que serão feitas. Mas há algumas conver-
gências interessantes e parece que cada campo
tem algo a aprender com os outros.

Como se pode notar, não há um consenso sobre o conceito de organização do conhecimento (independente do termo utilizado, organização ou representação) entre os autores. Em artigo recente, Bräscher e Café (2008), apresentam uma proposta conceitual preliminar para as áreas de organização da informação, organização do conhecimento, representação da informação e representação do conhecimento. As autoras se baseiam nos conceitos de Fogl sobre informação e conhecimento e nas definições citadas anteriormente de Taylor, Svenonius, Hodge e Dahlberg, além de outros autores como Shera, Egan e Soergel. Sobre organização e representação da informação, autoras concluem então que:

A organização da informação é, portanto, um processo que envolve a descrição física e de conteúdo dos objetos informacionais. $\bigcirc$ produto desse processo descritivo é a representação da informação, entendida como um conjunto de elementos descritivos que representam os atributos de um objeto informacional específico (Brascher; Café, 2008, p.5). 
E em relação à organização e representação do conhecimento:

Delineamos a organização do conhecimento
como o processo de modelagem do conheci-
mento que visa a construção de representações
do conhecimento. [...] A representação do co-
nhecimento é feita por meio de diferentes tipos
de sistemas de organização do conhecimento
(SOC) que são sistemas conceituais que repre-
sentam determinado domínio por meio da sis-
tematização dos conceitos e das relações se-
mânticas que se estabelecem entre eles
(Bräscher; Café, 2008, p.8).

Para fins desta análise, serão adotadas as abordagens em organização do conhecimento propostas por Hiørland e a proposta conceitual de Bräscher e Café, para organização do conhecimento, organização da informação, representação do conhecimento e representação da informação. Apenas a denominação das autoras para Sistemas de organização do conhecimento não será adotada, utilizando-se aqui, conforme explicado anteriormente, a denominação Esquemas de representação do conhecimento.

\section{Abordagens em organização do conheci- mento}

A partir da sistematização proposta por Hiørland (2007a), pode-se traçar um pequeno histórico das abordagens em organização do conhecimento. No final do século XIX, surgiram os primeiros sistemas de classificação bibliográfica, os enumerativos, como a CDD, proposta do Melvin Dewey em 1876. Hiørland (2007a) considera as classificações enumerativas como abordagens tradicionais em organização do conhecimento.

Já no século XX, surgiram outras abordagens, como o modelo facetado (analítico-sintético) de Ranganathan, contrapondo os princípios tradicionais da classificação enumerativa. A partir dos anos 1950, a introdução dos computadores trouxe uma grande mudança: o uso das máquinas para a recuperação da informação. Hiørland (2007d) considera a recuperação da informação como uma nova abordagem em organização do conhecimento. A criação do Science Citation Index, nos anos 1960, trouxe mais uma abordagem em organização do conhecimento, a abordagem bibliométrica. Já nos anos 1970 e 1980, a ênfase dada ao usuário gerou o desenvolvimento de abordagens cognitivas e baseadas nos usuários. Os anos 1990 foram marcados pela influência das novas tecnologias, como a possibilidade de buscas em texto completo e do modelo de Web Semântica. Essa fase é considerada por Hiørland (2007a) como uma continuação da abordagem em recuperação da informação. Ainda nos anos 1990, cresce o interesse em abordagens sociais, como a análise de domínio. Vale destacar ainda as tendências atuais em organização do conhecimento, que encontram bases na Arquitetura da Informação.

Entre as diversas abordagens, propõe-se agora explorar algumas específicas, nas quais, acredita-se, a folksonomia pode encontrar bases para ser analisada como um esquema de representação do conhecimento.

\section{Abordagem baseada no usuário}

A abordagem baseada no usuário surge nos anos 1970, auge dos estudos de uso e usuários. Segundo Hiørland (2007g), os sistemas de representação do conhecimento e seu processo de elaboração podem envolver, de uma forma ou de outra, dados fornecidos pelos usuários ou sobre os usuários. É importante fazer uma distinção básica entre abordagens baseadas em usuários e abordagens amigáveis (user-friendly): enquanto na primeira o sistema de representação é gerado a partir de dados fornecidos pelos usuários ou sobre eles, a segunda está relacionada com facilidade de uso, interatividade e capacidade de intuição do usuário no sistema. Nesse sentido, considera-se, por exemplo, que os sistemas de classificação bibliográfica como a CDU Classificação Decimal Universal (CDU) não são abordagens amigáveis, já que as notações que representam os assuntos não são intuitivas e confundem a maioria dos usuários.

Restringindo-se à abordagem baseada no usuário, apenas as "abordagens em Organização do conhecimento, que são principalmente baseadas nos dados obtidos dos usuários [...] $]^{3 \prime \prime}$ (Hiørland, 2007g,

\footnotetext{
${ }^{3}$ Approaches to $\mathrm{KO}$ that is mainly based on data obtained from users belong to the user-based approaches to $\mathrm{KO}$.
} 
tradução nossa), pode-se encontrar uma clara relação com a folksonomia, já que o processo de etiquetagem (tagging), que gera os termos do sistema, é totalmente realizado pelo usuário.

Outro aspecto considerado básico por Hiørland (2007g) nas abordagens orientadas ao usuário é que estas devem ter como foco a garantia do usuário em detrimento da garantia literária. Deve-se, então privilegiar, para a inclusão de termos no sistema, a terminologia do usuário e não a dos documentos/ objetos informacionais. Neste caso, a folksonomia também pode ser considerada: o próprio usuário adiciona o termo, garantindo seu uso posterior na recuperação da informação. Pode-se questionar o processo de escolha dos termos pelo usuário, já que este pode utilizar termos do próprio documento, como o título, por exemplo. Mas supõe-se que o usuário só fará desta forma se o termo do documento coincide com aquele de seu uso habitual.

Desta forma, entende-se que a folksonomia encontra bases nesta abordagem para ser considerada um esquema de representação do conhecimento, já que possui duas das principais características da abordagem baseada no usuário.

\section{Abordagem cognitiva}

A abordagem cognitiva em Biblioteconomia e Ciência da Informação surgiu como paradigma para a Organização do conhecimento a partir de 1992, quando a Segunda Conferência Internacional da International Society for Knowledge Organization (ISKO) teve essa abordagem como tema. Hiørland (2007b) faz uma crítica ao editorial escrito por Dalhberg sobre a visão cognitiva em Organização do conhecimento, pois o ensaio traz a visão cognitiva como uma tautologia: todas as abordagens em Organização do conhecimento devem se preocupar, de uma forma ou de outra, com questões conceituais e cognitivas. Segundo Hiørland (2007b), muito pouco é realmente dito sobre a visão cognitiva em Organização do conhecimento nesse artigo. $\bigcirc$ autor afirma ainda que, apesar de algumas discussões sobre o assunto, não há uma avaliação sobre o que a visão cognitiva pode trazer para o campo da Organização do conhecimento.
Aparentemente, não há um consenso entre os autores da área.

A visão cognitiva é também relacionada ao comportamento de busca do usuário: "Paradigmas cognitivos indicam o comportamento de busca de conhecimento de indivíduos e grupos de indivíduos ${ }^{4 "}$ (Hjørland, 2007b, tradução nossa). É sugerido ainda que a análise de tal comportamento de busca possa fornecer diretrizes para a organização da informação em base de dados e ambientes similares.

Um conclusão muito citada sobre o significado da visão cognitiva é a de De Mai, em 1980, citado por Hiørland (2007b, tradução nossa): "um sistema de recuperação de informação deve refletir em suas operações, de uma forma ou de outra, o mundo cognitivo do usuário". Esse mundo cognitivo do usuário não deve ser melhor representado do que se gerado pelo próprio usuário. De forma semelhante, Foskett (1980) afirma que "o serviço de informação mais eficaz é aquele que é projetado especificamente para cada usuário, baseado em suas necessidades conhecidas." Levando-se em conta esses autores, tem-se, então, uma forte base para a folksonomia - acredita-se que nenhuma outra forma de representação do conhecimento está tão diretamente ligada ao mundo cognitivo e às necessidades do usuário.

Sobre esse aspecto, Hiørland (2007b) afirma ainda que a representação do conhecimento não é somente tentar criar representações tão objetivas quanto possíveis, mas otimizá-las em relação a certas tarefas e valores. Neste caso, a folksonomia também pode ser citada: o usuário descreve o objeto de informação de forma a facilitar uma tarefa futura, além de fazer um julgamento de valor, o que não necessariamente corresponderia à forma mais objetiva.

Em artigo recente, Sinha (2005) faz uma análise cognitiva de processo de etiquetagem e como seu baixo custo o torna popular. A autora descreve como se dá tal processo, além de comparar a etiquetagem com a categorização, em termos cognitivos. A Figura 1 mostra como ocorre o processo de etiquetagem no nível cognitivo. $\bigcirc$ estágio zero corresponde à seleção de um item (objeto de informação) que vale a pena ser recuperado num momento posterior. $\bigcirc$ próximo estágio é um processo de comparação de similaridade entre o item e os conceitos candidatos a virarem etiquetas.

\footnotetext{
${ }^{4}$ Cognitive paradigms indicate the knowledge seeking behaviour of individuals and groups of individuals.
} 
Nessa fase, conceitos semânticos relacionados são ativados: tanto conceitos gerais quanto os mais pessoais a que o objeto remete e ainda características físicas podem ser lembradas, segundo estudos da psicologia cognitiva. A partir do momento que esses conceitos são ativados, externalizá-los, escrevendo-os na forma de etiquetas é bastante simples: não há um processo de filtragem ou julgamento nesse estágio, pode-se usar tantas associações quanto necessário. Sinha (2005) afirma que é dessa forma que o processo de etiquetagem funciona do ponto de vista cognitivo.

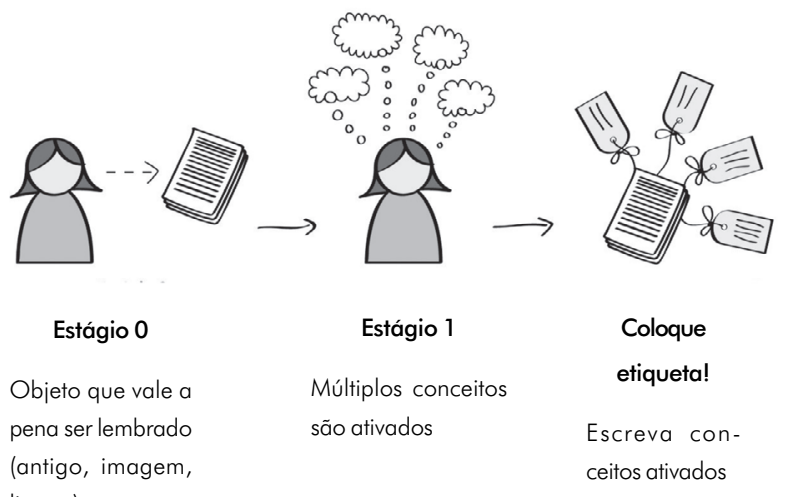

Figura 1. Processo cognitivo por trás da etiquetagem.

Fonte: Adaptado de Sinha (2005), tradução nossa.
Segundo a mesma autora, já o processo de categorização, ilustrado pela Figura 2, inclui uma nova fase, que é o processo de decisão. As categorias candidatas devem ser julgadas, e decide-se, então, qual é a correta para descrever dado item. Trata-se de um processo cognitivo básico: categorizamos as coisas o tempo todo, de forma quase automática.

O estágio que dificulta o processo de categorização, segundo a autora, é por ela denominado post-activation analysis paralysis, algo como uma parada para análise, que, na figura, corresponde ao estágio 2. Uma das dificuldades se relaciona com a questão cultural: falta consenso cultural no ambiente digital, e a categorização é com frequência baseada em conhecimento cultural. Além disso, a autora afirma que, no ambiente digital, a etiquetagem não só fornece a categorização de um objeto, como também aumenta sua recuperabilidade (findability). Dessa forma, devese considerar não só a categoria mais adequada, mas também a categoria com maior probabilidade de se encontrar o item no momento da busca. Essas duas questões levam a respostas conflitantes e complicam o processo de categorização, pois se deve considerar ainda o esquema de categorização maior em que se insere a informação (Sinha, 2005).

estágio de analysis-paralysis, segundo a autora, corresponde ao temor de se tomar uma decisão errada ao se categorizar um objeto, o que implicaria

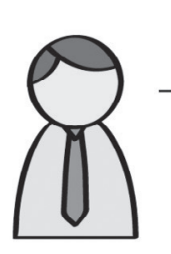

Estágio 0

Objeto que vale a pena ser lembrado (antigo, imagem, livro...)
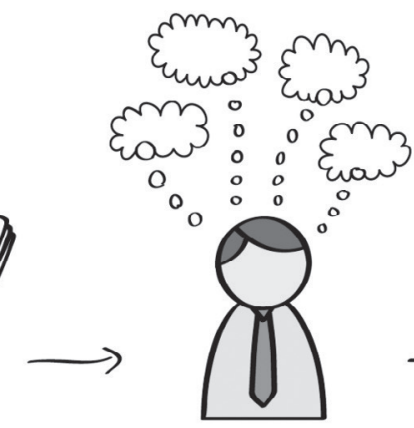

Estágio 1

Múltiplos conceitos são ativados
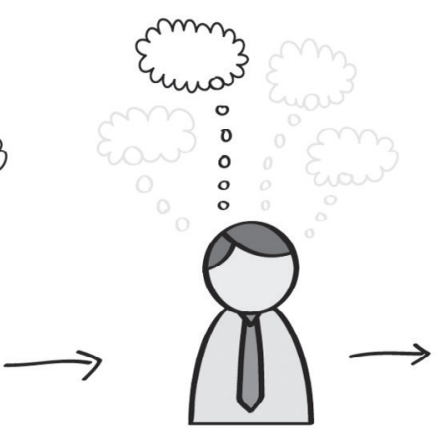

Estágio 2

Escolha UM dos conceitos ativados

Parada para análise

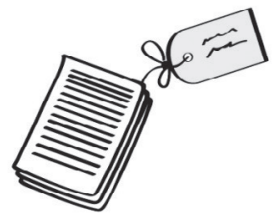

Categorize!

Anote o conceito escolhido

Figura 2. Processo cognitivo por trás da categorização.

Fonte: Adaptado de Sinha (2005), tradução nossa. 
na perda do item em termos de recuperabilidade. É justamente essa parte da decisão (escolha da categoria correta) que é eliminada no processo de etiquetagem. Em suma: o custo cognitivo do processo de etiquetagem é bem mais baixo.

É importante lembrar que, para a organização do conhecimento, o item teórico mais essencial é o fato de que a organização do conhecimento deve ser baseada em unidades do conhecimento - que são os conceitos (Dahlberg, 1993). Nas folksonomias, as etiquetas (tags) representam termos, mas o que é ativado no processo cognitivo visto nas Figuras 1 e 2 são os conceitos, os seja, a estrutura é construída a partir dos conceitos fornecidos pelos usuários.

A partir desta análise do processo cognitivo, conclui-se que a etiquetagem é relativamente simples sob esse ponto de vista. Tal fato deve contribuir para a expansão de folksonomias no ambiente digital. A abordagem cognitiva é, portanto, relevante para a análise da folksonomia.

\section{Organização social do conhecimento}

Segundo Hiørland (2007e) a organização social do conhecimento se contrapõe à organização intelectual do conhecimento. A primeira se refere à organização do conhecimento - categorização das disciplinas, por exemplo - feita de acordo com interesses institucionais e acadêmicos, enquanto a segunda se refere à organização do conhecimento com bases mais inerentes ao próprio conhecimento, ou seja, à área do saber em questão. $\bigcirc$ autor afirma que esta distinção é fundamental para a teoria da organização do conhecimento, e que não está relacionada apenas à organização das ciências, mas do conhecimento em geral.

A organização social não chega a ser uma abordagem de Organização do conhecimento, mas uma vertente em que se podem apoiar várias abordagens, como, por exemplo, a bibliométrica. Nesse sentido, a discussão aqui levará em conta a organização social do conhecimento como aquela feita pelo homem, com base em seus princípios e diretrizes (e gerada por uma determinada comunidade), e não com base no próprio conhecimento do domínio. É, portanto, uma forma ainda mais arbitrária de representação do conhecimento - já que todas são em algum grau.
Posto isso, tem-se, então, que a folksonomia como representação do conhecimento gerada pelo usuário, ou por comunidades afins, pode-se ser dita como uma forma de organização social do conhecimento: arbitrária, baseada nos princípios dos próprios usuários e compartilhada num meio social determinado. Tal visão pode ser analisada a partir do artigo de Sinha (2006), que após uma análise cognitiva do processo de etiquetagem, fez uma análise social do mesmo.

Sinha (2006) afirma que a formação social básica permitida pela etiquetagem está mais para "multidão" que para um verdadeiro grupo: os usuários não se conhecem e não têm nenhum tipo de contato uns com os outros. $\bigcirc$ processo de etiquetagem levaria então a uma "sabedoria das multidões" - conceito defendido por James Suroweick. Para Sinha (2006), o processo de etiquetagem estaria livre dos aspectos negativos de comportamentos de "multidão", e os quatro princípios da "sabedoria das multidões" estão presentes: diversidade de opiniões, independência dos membros, descentralização e método de agregar opiniões. A Figura 3 ilustra a interação social e a transmissão conceitual com etiquetas.

Pode-se ressaltar ainda que na própria denominação do processo que gera a folksonomia está inserido o conceito "social": classificação social, etiquetagem social, categorização social. Conclui-se então que a folksonomia, se considerada como um esquema de representação do conhecimento, estará inserida nessa vertente da organização social do conhecimento.

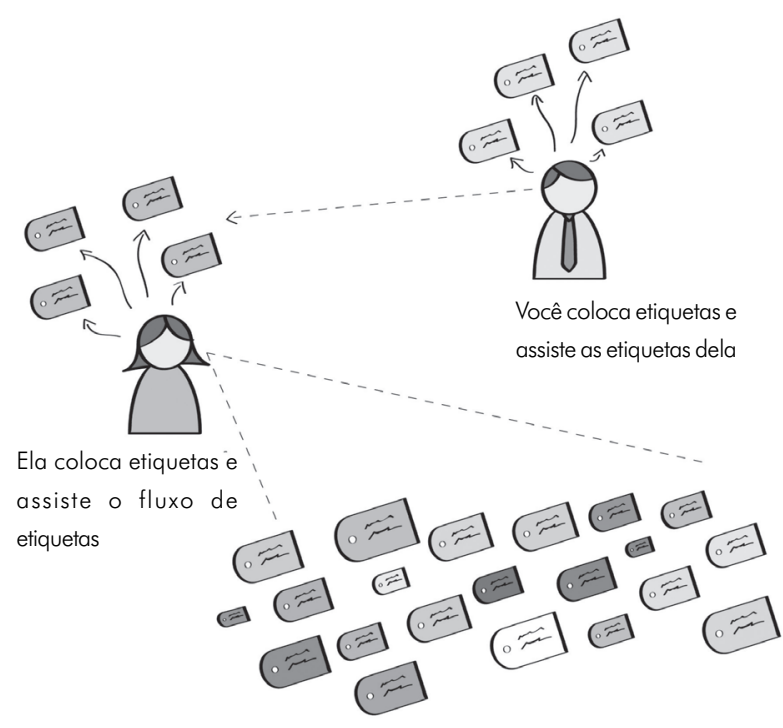

Figura 3. Interação social e transmissão conceitual de etiquetas. Fonte: Adaptado de Sinha (2005), tradução nossa. 


\section{Abordagem da recuperação da informação}

Entre as abordagens da Organização do conhecimento discutidas por Hiørland (2007d), encontra-se a abordagem da Recuperação da Informação (RI). Inicialmente, o autor questiona se a RI pode ser caracterizada também como uma abordagem de Organização do conhecimento, já que normalmente ela é considerada como uma sub-disciplina da Biblioteconomia e Ciência da Informação.

A recuperação da informação é baseada no pressuposto fundamental da correspondência entre a questão do usuário e a representação do documento que é o princípio dos mecanismos de busca. Há algumas fraquezas em relação a esse conceito. Pode-se, por exemplo, considerar relevante identificar co- citações em artigos, independentemente dos documentos serem ou não similares. Neste caso, uma simples "transformação da pergunta" não resolveria (Hiørland, 2007d).

Sendo assim, de acordo com Hiørland (2007d), deve-se questionar os resultados obtidos por essa abordagem. Caso contrário, não seria mais necessário - campo de pesquisa em Organização do conhecimento, pois este poderia ser substituído por Recuperação da informação. Por esta razão, o autor considera importante considerar a RI entre as abordagens em Organização do conhecimento, para que se possam identificar assim seus pontos fortes e fracos.

Considerando-se então a Rl como uma abordagem em Organização do conhecimento, pode-se identificar como a folksonomia se encaixaria nessa abordagem.

O que se pode considerar da folksonomia para a abordagem de Rl é a chamada etiquetagem implícita. Trata-se de usar as próprias questões de busca (queries) como etiquetas para os documentos recuperados. Em artigo recente, Morisson (2007) faz uma análise das razões que levam os usuários a etiquetarem. Entre as encontradas pelo autor está a etiquetagem implícita: "os usuários colocam etiquetas incidentalmente enquanto executam outras tarefas de recuperação da informação" (Morisson, 2007, p.14), ou seja, os usuários colocam etiquetas sem nem saber que estão fazendo isso. Tal processo ocorre quando um sistema de informação utiliza o texto da busca e o associa ao documento recuperado escolhido pelo usuário. $\mathrm{O}$ problema encontrado nesse método é quando um documento não foi descrito por um termo que é usado pelo usuário para buscá-lo. Assim, a interseção entre pergunta e resultado não ocorrerá, e a etiqueta usada nunca será aplicada a determinado documento. A solução encontrada pelo autor para este caso é adicionar ele mesmo a etiqueta manualmente no sistema.

Como a abordagem da Rl se baseia na relação entre a pergunta do usuário e a resposta do sistema, a etiquetagem implícita seria utilizada da mesma forma: a pergunta do usuário seria aproveitada como etiqueta para um objeto informacional.

Pode-se dizer então que, quando desta forma, a etiquetagem - processo que gera a folksonomia, guarda relações estreitas com abordagem de RI. Neste processo, o usuário participa da categorização dos recursos indiretamente e sem saber que o está fazendo, o que, acredita-se, afastaria a folksonomia da visão cognitiva.

\section{Folksonomia como esquema de representação}

Após a análise das características da folksonomia comuns às abordagens da organização do conhecimento, pode-se agora analisar seus aspectos positivos e negativos para, então, chegar-se a uma definição da questão proposta inicialmente.

Dentro das características que permitem incluir a folksonomia nas abordagens em Organização do conhecimento, podem ser encontrados pontos positivos e negativos. O fato de a folksonomia ser construída a partir de dados obtidos dos próprios usuários é algo positivo no sentido da garantia do uso, ou seja, o termo usado para representar o documento será o mesmo usado para recuperá-lo posteriormente, por determinado usuário. Neste caso, a garantia de uso do termo na literatura (garantia literária), parece não importar muito, já que a folksonomia é construída a posteriori - não há uma etapa de análise dos documentos do domínio para então se coletar termos e criar posteriormente a base para 0 esquema de representação, como no caso dos tesauros, por exemplo.

Dentro das abordagens orientadas aos usuários, é interessante a discussão feita por Broughton et al. (2005) sobre o que é organizado em cada abordagem, 
ou seja, o que é considerada como unidade a ser organizada. Para esses autores, nas abordagens cognitivas e orientadas a usuários, a unidade organizável é a estrutura cognitiva, o individual. Nessa perspectiva, a folksonomia poderia certamente ser considerada uma estrutura de representação do conhecimento.

Apesar disso, o uso da estrutura cognitiva como unidade organizável pode ser questionada. $\mathrm{Na}$ abordagem cognitiva, a mesma característica pode ser considerada positiva e negativa: o mundo cognitivo do usuário. Representar o mundo cognitivo do usuário é algo que nenhum outro tipo de representação pode fazer tão bem quanto a folksonomia - ninguém melhor que o próprio usuário para representar seu mundo cognitivo. É sabido que um esquema de representação do conhecimento tão mais preciso será quanto mais restrito e específico for o domínio representado. Levando-se essa especificação ao extremo, deve-se chegar ao usuário, a uma única pessoa - seu mundo cognitivo é certamente o extremo de uma especificação de conhecimento. Sendo assim, a representação do conhecimento gerada numa folksonomia teria uma grande vantagem. Porém, para apenas um usuário:

\footnotetext{
Pessoas colocando etiquetas para seu próprio uso (ou reuso) selecionam palavras que usam e acreditam que entendem. Essa perspectiva significa que as etiquetas estão corretas para pelo menos uma pessoa e nem sempre para uma comunidade ou disciplina a que pertence (Wal, 2005, online).
}

A representação do conhecimento fica desta forma, atomizada, o que suscita a questão da utilidade dessa representação. Além disso, a grande vantagem do mundo cognitivo do usuário é diluída à medida que mais e mais usuários colaboram com suas tags em um serviço que vai gerar uma folksonomia.

De qualquer forma, acredita-se que a representação gerada em cada serviço deverá trazer, em algum grau, algo válido para determinada comunidade de usuários que a gerou. Os usuários de determinados serviços de informação na web podem ter interesses comuns e até mesmo formar essas comunidades dentro dos serviços: "a web se auto-organiza de tal forma que a estrutura de links permite uma identificação eficiente de comunidades" (Flake et al., 2002, p.66). Acredita-se que a etiquetagem é um processo que contribui nessa auto-organização da web.

Em todo caso, haverá sempre um trade off nos esquemas de representação do conhecimento: quanto mais específicos e próximos da cognição, menor sua utilidade para um grande número de usuários.

A característica de classificação social encontrada no modelo de organização social do conhecimento traz os mesmos problemas dos outros sistemas gerados nesta mesma vertente: a arbitrariedade nas decisões. Apesar disso, a folksonomia tem a vantagem de ser um esquema botton-up, em que os termos são gerados a partir do mais específico, não passando pela fase da categorização, como visto anteriormente. Além disso, apesar dos termos serem gerados pelos próprios usuários, estes o fazem de uma forma um pouco mais independente que um determinado grupo social escolhido para organizar um domínio, como no exemplo de Hjørland (2007e) da categorização de disciplinas em uma universidade. Os usuários que geram as folksonomias podem ser influenciados pelas tags de outros usuários, mas acredita-se que o grau de parcialidade e as questões políticas são menores que num grupo que, de fato, se conhece e se reúne para elaborar uma categorização de um domínio.

Por fim, a etiquetagem implícita encontrada na abordagem da Recuperação da informação apresenta como vantagem o uso das próprias perguntas de busca (queries) como tags para os objetos informacionais, sem que o usuário mesmo saiba. Isso por um lado é bom, pois muitas vezes o usuário de um sistema de informação que disponibiliza a ferramenta de etiquetagem não usa o recurso, ou seja, não contribui para gerar a folksonomia naquele sistema. Já na etiquetagem implícita, o usuário executa uma tarefa comum de busca da qual necessita e nem fica sabendo que está colaborando para o sistema de etiquetagem dispensa-se assim a "boa vontade" do usuário. A parte falha é que nem todo objeto de informação poderá ser etiquetado dessa forma, pois alguns tipos de rótulos usados pelos usuários não serão encontrados no recurso.

\section{Como esse esquema reflete ou falha em refletir conhecimento}

A partir do exposto anteriormente, tem-se então que o processo de etiquetagem gera uma folksonomia, 
e esta pode ser considerada uma representação do conhecimento, no sentido de, no mínimo, refletir o conhecimento de determinada comunidade. Assim, a representação gráfica usual das folksonomias - a tag cloud (Figura 4), reflete os assuntos mais recorrentes em determinado momento e em determinado sistema. Dessa forma, pode-se dizer que a folksonomia pode ser usada como forma de descoberta de conhecimento: a partir da análise das tags mais frequentes é possível determinar tendências e interesses de comunidade analisada.

As falhas mais citadas do processo de etiquetagem são as de falta de padronização: na maioria dos casos, não há regras para a aplicação de etiquetas. Desta forma, o vocabulário é totalmente livre, gerando os conhecidos problemas que costumam ser resolvidos por um vocabulário controlado.

Entende-se que a folksonomia, comparada aos outros esquemas de representação do conhecimento, é gerada de forma inversa: primeiro se classificam os objetos informacionais, e, posteriormente, surge uma folksonomia, representada visualmente pela tag cloud. Já nas outras ferramentas como as taxonomias e os tesauros, os objetos informacionais são classificados somente quando elas já existem: um tesauro, por exemplo, é usado para a escolha dos termos que irão descrever o conteúdo de um documento (Figura 5).

Ou seja, a folksonomia é construída a posteriori em relação aos outros esquemas. Isso traz algumas falhas para a representação do conhecimento, como observado por Sinha (2006), o surgimento de termos sem conceito, já que não há uma formalização ou um consenso explícito da comunidade envolvida.

Assim, pode-se entender que a folksonomia estaria inserida nas duas vertentes de Organização do

art austrália baby beach birthday blue bw califórnia canadá canon cat china

christmas city dog england europe family festival flower flowers food france

friends fun germany holiday índia italy japan london me méxico music nature

new newyork night nikon nyc paris park party people portrait red sanfrancisco

sky snow spain summer sunset taiwan travel trip uk usa vacation water

wedding winter

Figura 4. Exemplo de tag cloud: Flickr em 15/6/2008. conhecimento propostas por Broughton et al. (2005): a dos processos de organização e a dos sistemas de organização do conhecimento. Sobre esse aspecto, alguns autores como Trant (2006) fazem uma clara distinção entre folksonomia e social tagging (etiquetagem). Neste caso, a etiquetagem estaria na vertente dos processos de organização (como forma de indexação colaborativa) e a folksonomia na dos sistemas de organização do conhecimento.

\section{CONSIDERAÇÕES FINAIS}

A proposta deste artigo foi analisar a folksonomia em cada uma das abordagens propostas e seus pontos fortes e fracos como esquema de representação do conhecimento. Feito isso, tem-se então uma base que permite enquadrar a folksonomia nos esquemas de representação do conhecimento, ao menos em certo sentido. Acredita-se, assim, que as características da folksonomia encontradas nas abordagens propostas possam levar a tal conclusão.

Para uma definição formal maior, propõe-se um estudo que identifique o que faz com que um modelo seja considerado um esquema de representação do conhecimento, ou seja, quais os requisitos formais básicos que permitem tal definição. Sugere-se que se identifiquem esses requisitos nos esquemas tradicionais, como tesauros, taxonomias e ontologias, e, a partir daí, se faça uma comparação com as características da folksonomia.

De qualquer forma, acredita-se que esta análise pode servir como contribuição para o entendimento das folksonomias e sua contextualização na teoria da Organização do conhecimento.

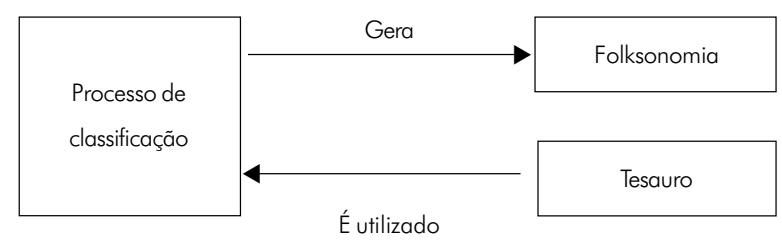

Figura 5. Processo inverso: folksonomia x instrumentos tradicionais. 
BRASCHER, M.; CAFÉ, L. Organização da informação ou organização do conhecimento? In: ENCONTRO NACIONAL DE PESQUISA EM CIÊNCIA DA INFORMAÇÃO, 9., 2008, São Paulo. Anais... São Paulo: USP, 2008.1 CD-ROM.

BROUGHTON, V.; HANSSON, J.; HJØRLAND, B.; LÓPEZHUERTAS, M.J. Knowledge Organization. Report of working group. In: LIS-education in Europe. Working Seminar, at the Royal School of Library and Information Science. Available from: <http:// www.db.dk/bh/lifeboat_ko/HISTORY\%20\&\%20THEORY/ LIS\%20Education\%20in\%20EuropeDraft_KO_Submitted.htm > . Cited: 8 Jul. 2008.

DALHBERG, I. Knowledge organization: its scope and possibilities. Knowledge Organization, v.20, n.4, p.21 1-222, 1993.

FOSKETT, D.J. Psicologia do usuário. In: CONTRIBUIÇÃO da psicologia para o estudo dos usuários da informação técnicocientífica. Rio de Janeiro: Calunga, 1980. p.11-29.

HJØRLAND, B. Approaches to knowledge organization (Theories of KO). 2007a. Available from: <http://www.db.dk/bh/ lifeboat_ko/HISTORY\%20\&\%20THEORYapproaches_to knowledge_organiza.htm>. Cited: 26 May 2008.

HJØRLAND, B. Cognitive views in knowledge organization. 2007b. Available from: <http://www.db.dk/bh/lifeboat ko/ HISTORY\%20\&\%20THEORY/cognitivein_knowledge orga.htm>. Cited: 1 Jun. 2008.

HJØRLAND, B. Discussion of Dahlberg's theory of concepts and knowledge organization (KO). 2007c. Available from: < http:// www.db.dk/bh/lifeboat_ko/CONCEPTS/discussion_of_dahlberg. htm > . Cited: 24 May 2008.

HJØRLAND, B. Information retrieval (IR) as challenger to knowledge organization. 2007d. Available from: <http:// www.db.dk/bh/lifeboat ko/HISTORY\%20\&\%20THEORY/ information retrieval\%20approach.htm >. Cited: 7 Jun. 2008

HJØRLAND, B. Knowledge organization systems. 2008. Available from: <http://www.db.dk/bh/lifeboat_ko/CONCEPTS/ knowledge_organization_systems.htm>. Cite: 27 May 2008.

HJØRLAND, B. Social organization of knowledge. 2007e. Available from: <http://www.db.dk/bh/lifeboat ko/CONCEPTS/ social_organization_of_knowledge.htm > . Cited: 26 May 2008.

HJØRLAND, B. "Traditional approaches" to knowledge organization. 2007f. Available from: <http://www.db.dk/bh/ lifeboat_ko/HISTORY\% $20 \& \% 20$ THEORY/ traditional approaches to knowle.htm>. Cited: 26 May 2008.

HJØRLAND, B. User and user studies in knowleadge organization. 2007g. Available from: <http://www.db.dk/bh/
lifeboat_ko/CONCEPTS/user_and_user_studies.htm > . Cited: 31 May 2008.

HODGE, G. Systems of knowledge organization for digital libraries: beyond traditional authority files. Washington, DC: the Council on Library and Information Resources, 2000. Available from: <http://www.clir.org/pubs/reports/pub91/contents.html>. Cited: $15 \mathrm{Jul} .2008$.

FLAKE, G.W. et al. Self-organization and identification os web communities. IEEE Computer. v.35, n.3, p.66-71, 2002.

MORRISON, P.J. Why are they tagging, and why do we want them to? Bulletin of the American Society for Information Science and Technology, p. 12-15 Oct./Nov. 2007.

O'REILLY, T. What is Web 2.0. 2005. Available from: <http:// www.oreillynet.com/pub/a/oreilly/tim/news/2005/09/30/what-isweb-20.html?page =1 > . Cited: 16 May 2008 .

SINHA, R. A cognitive analysis of tagging (or how the lower cognitive cost of tagging makes it popular). 2005. Available from: <http://rashmisinha.com/2005/09/27/a-cognitive-analysis-oftagging/\#more-1 12>. Cited: 2 Jun. 2008.

SINHA, R. A social analysis of tagging (or how tagging transforms the solitary browsing experience into a social one). 2006. Available from: <http://web.archive.org/web/20060130101032/http:// www.rashmisinha.com/archives/06 01/social-tagging.html >. Cited: 6 Jun. 2008.

SOWA, J.F. Knowledge representation: logical, philosophical, and computational foundations. Pacific Grove, CA: Brooks Cole Publishing, 2000. Available from: <http://www.jfsowa.com/ krbook/index.htm>. Cited: 15 Jul. 2008.

TAYLOR, A. The organization of information. $2^{\text {nd }}$ ed. [s.I.]: Library and Information Science Text Series, 2003.

TRANT, J. Exploring the potential for social tagging and folksonomy in art museums: proof of concept. New Review of Hypermedia and Multimedia, v.12, n.1, 2006. Available from: $<$ www.archimuse.com/papers/steve-nrhm-0605preprint.pdf > . Cited: 17 Jul. 2008.

VICKERY, B. C. Knowledge representation. Journal of Documentation, v.42, n.3, 1986, p.145-159, 1986.

WAL, T.V. Tagging for fun and finding. 2005. Available from: <http://www.ok-cancel.com/archives/article/2005/07/taggingfor-fun-and-finding.html>. Cited: 28 Jun. 2009.

WAL, T.V. Folksonomy. 2007. Available from: <http:// www.vanderwal.net/folksonomy.html>. Cited: 16 May 2008. 
